\title{
Emergency communication network design and key technology research
}

\author{
Jin Meng ${ }^{1, a}$, Hongyan Liu ${ }^{1, b}$, Xiao Zhang ${ }^{1, c}$, Mingshun Xing ${ }^{1, c}$, Hui Li $^{1, c}$ \\ ${ }^{1}$ Xi'an Communication Institute, Xi'an 710106, China \\ a51375908@qq.com, b813086903@qq.com, c23414388@qq.com
}

\begin{abstract}
Keywords: emergency communication network; Ad hoc network; wireless sensor network; satellite communication

Abstract: According to the characteristics and requirements of emergency communication, this paper presents a scheme of emergency communication network deployment, and illustrates the organization mode of communication network in the network structure. In addition, the key technologies of emergency communication network are discussed, in order to provide reference for the establishment of emergency communication network in China.
\end{abstract}

China is one of the countries with frequent natural disasters. Major natural disasters often lead to public communication network failure. when the existing public communication network cannot satisfy the normal emergency communication requirements, emergency communications will be in great demand to provide communications security for rescue operations [1]. Emergency communication is one of the urgent tasks for responding to natural or man-made urgent emergency with a nonconventional means of communication to ensure the release of disaster relief command, important information transmission and rescue command completion [2]. Emergency communication is not a new communication technology, but an integrated use of all kinds of communication means and methods in case of emergency. Its core is to comprehensively consider all factors of emergency nature, geographical environment and climatic conditions, and then choose the most reasonable communication resources as an emergency measure.

\section{Main features of emergency communication network}

After the occurrence of natural disasters and other unexpected emergency, the emergency procedure usually experiences four main stages supported by emergency communication system, namely, the emergency alarm, emergency processing stage, stage of emergency notification and emergency communication [3]. The network types and key technologies used in each phase are different ones.

Emergency communication networks often need to operate in complex and harsh environment, the geographical and climatic conditions are varied, the network size can be different. This network has the following salient features: involving a variety of network system with different abilities and characteristics, such as cable transmission network, wireless sensor network, cellular mobile communication network, the air relay system and satellite networks; contains a large number of mobile speed and the ability to deal with different communication unit and coordinate communication; includes a wide range of business, with asymmetric information transmission characteristics, such as information received by rescue workers at the scene are more often than sent ones.

\section{Emergency communications network deployment}

Emergency communication systems must be effective and comprehensive in the inspection of deployment area, in order to prevent and manage possible risks. A number of vehicle power station and a large number of portable low power radio are typically deployed in the on-site emergency, which is decided by emergency communication needs, such as the incident area, the terrain, the station's processing ability and power transmission and wireless channel quality and other factors. The vehicle platform has a greater power and stronger processing capacity, as the backbone node; 
the power and processing capacity of the portable radio station is weak, which is a common user node. Backbone Network Mobile (MBN) can be interconnected by a plurality of vehicular stations which can be interconnected by a wire or a microwave relay, and a portable radio station can be used to access the mobile trunk network, and can be connected with each other to form a user network.

In order to take into account the performance and scalability of the network, the emergency communication network adopts hierarchical clustering network structure [3]. In the heterogeneous emergency communication network, strong function of the backbone nodes generally act as the cluster head, which usually consists of a backbone node and its direct communication with user nodes and user nodes in the cluster is related to the number of user nodes transmit power, a backbone node capacity, terrain, the channel propagation characteristics and user nodes distribution. As a common node, it can be selected as the cluster head, which is closer to the node and the light load.

Emergency scene command center CCU should first be deployed in a position to facilitate coordination and command, and then through the backbone network to collect the situation of the incident area, and the CCU should be responsible for coordinating the communication between the rescue units. At the same time, an appropriate number of backbone nodes (CN) are deployed in a number of preferred locations, and the collections between the CNs or the CN and CCU are allowed. $\mathrm{ON}$ is connected to the $\mathrm{CN}$ by the way of self organization. Wireless ad hoc network topology often changes, so the requirements of the network protocol can quickly respond to the change, according to the changes of nodes and links in a timely manner to adjust. When there is available ON near the $\mathrm{CN}$, the $\mathrm{CN}$ can be used as the cluster head; otherwise, ON uses a cluster head election algorithm to select cluster head for networking. Cluster network structure has good scalability, which is easy to maintain and manage [3]. The backbone network made by the backbone nodes is mainly responsible for long-distance business transmission, small ad hoc networks are mainly responsible for the collection, transmission and reception of emergency site information. The on-site emergency communication network has a strong mobility, which means both ordinary nodes and backbone nodes can be moved, but the average speed of the backbone nodes is relatively slow. In order to meet the requirements of network nodes, the backbone nodes make adjustments according to the nodes the number and distribution of subordinate position. Therefore, the distribution of the common nodes determines the location of the backbone nodes and the topology of the main network.

When the emergency communication network is large, it is necessary to divide the whole network into a number of emergency response area networks. Each area network coverage of up to several $\mathrm{km}$ to dozens of $\mathrm{km}$, protecting a certain number of backbone nodes and providing communication services to ordinary nodes in the area. Communication between the regional network can be completed by the ground link network or air network. In order to improve the quality of communication and facilitate the management, the common nodes in each area network are expected to hop into the trunk network as far as possible. Sufficient number of backbone nodes can improve the robustness and service of the network, but it will increase the cost of network deployment. Therefore, it is necessary to make a reasonable configuration choice according to the communication needs. A feasible strategy: the number of backbone nodes should enable the backbone network to cover all the normal nodes, so that command and control and other important information can be rapidly transported to the ordinary nodes, allowing ordinary nodes to access backbone network in one hop or multi hop, reducing the number of backbone nodes and increase the system flexibility.

The emergency communication system using the IP protocol as a link connecting heterogeneous networks, including rear command center in a wired LAN, on-site emergency (wireless) emergency communication network, and rear command post and emergency communication transmission network (Internet, special cluster network and satellite communications network). The deployment of emergency communication network is shown in Figure 1. 


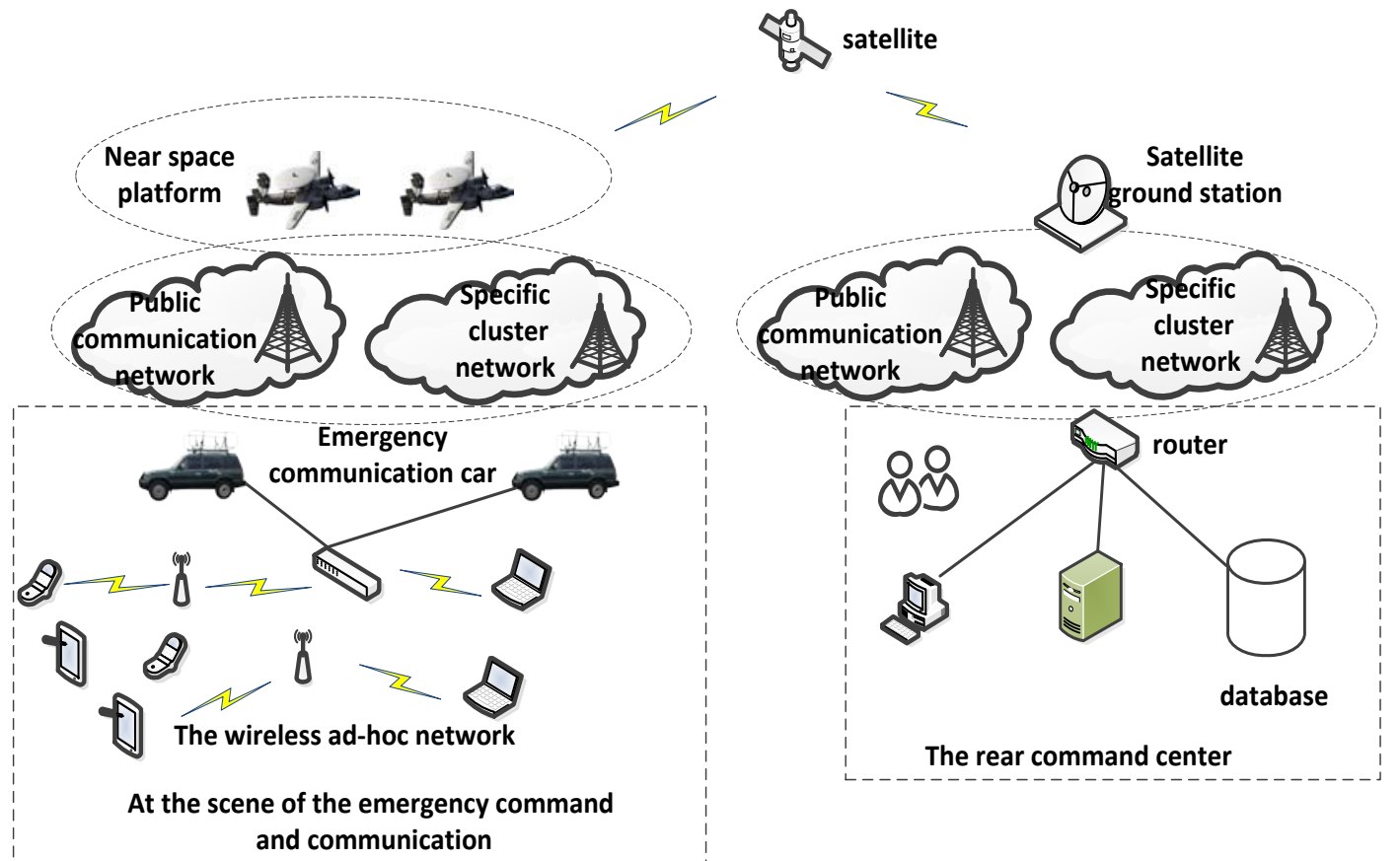

Figure 1 The deployment of emergency communication network

\section{Main technical means of emergency communication}

The emergency field communication technology should be based on wireless mode. At this stage, the main emergency response system has the following methods.

\section{- Satellite communications emergency response system}

The satellite communication system is a communication system which is not affected by the earth disaster, and it covers a wide area. But its high cost of use is difficult to be universal in the global personal mobile communications. The current application for the public is mainly for the transmission of television signals. For major natural disasters is directly related to the loss of state property and people's life safety, the satellite emergency communication system free from the influence of geographical environment is still one of the main means at the present stage of emergency communication network, which is of unique inportance.

\section{- Ad hoc mobile networks}

Ad hoc mobile network is also a kind of mobile ad hoc network, all nodes in the network composed of mobile hosts, when any 2 mobile hosts can communicate with each other in the communication range. At the same time, the mobile host also has the function of the router, it can realize the remote data transmission through the routing function of the mobile host. Ad hoc network has features as self-organization, network node independence, dynamic network topology, high-speed mobile environment fast networking [4], making it particularly suitable for the formation of disaster emergency data transmission network. Self-organizing network node can transmit the on-site scene where people find difficult to reach to commanders directly.

\section{- Cluster communication emergency system}

Cluster communication system is a kind of multi purpose and high efficient wireless dispatching communication system, which can be used for sharing resources, channels, equipment and services, and can dynamically distribute the limited channel to all users in the system. At the same time, based on the emergency communication system of trunking communication support group call, the PTT (push to talk, PTT) way quickly realize "one to many" call, so it is very suitable to carry out 
emergency command scheduling and trunking communication system and flexible networking, convenient, and is also an ideal means of communication to build the scene of the accident emergency command and dispatch platform.

\section{- Microwave communication emergency system}

Microwave communication system works in microwave band,its diffraction of the wireless signal is weak and its direct way of relay communication is based on the microwave station to avoid the mountains, buildings and other obstacles in the barrier, so to build microwave communication system on the location of the accident have certain requirements on the topography. However, emergency communication system based on microwave communication has the advantages of wide frequency band, large capacity and communication quality good, which can simultaneously transmit multiple emergency information (voice, data, fax, and video) and large capacity communication at the scene of the accident with the outside world.

\section{- Wireless Sensor Networks}

Wireless sensor networks (wireless sensor networks, WSN) is a self-organizing wireless network made by a group of wireless sensors, the information in regional geography can be sensed, acquired and processed by the network, and sent through the wireless network to the command center, for quick access to the scene of the accident information is of great significance. In recent years, due to the flexible networking, long data transmission distance, low cost features of wireless sensor network, it has seen rapid development in emergency communication field. It has become one of the optional technical means in emergency communication network, for it can work where there is wiring difficulties. .

\section{Conclusion}

The emergency communication network should make full use of the function, and it must make full use of all kinds of communication technology and comprehensive utilization of all kinds of existing network infrastructure and temporary deployment of network facilities. According to the characteristics and requirements of emergency communication, this paper presents a scheme of emergency communication network deployment, and illustrates the organization mode of communication network in the network structure. In addition, the key technologies of emergency communication network are discussed, in order to provide reference for the establishment of emergency communication network in China.

\section{Reference}

[1] Fubler H, Widmer J, Kasemann M. Contention-Based Forwarding for Mobile Ad Hoc Networks [J]. Elsevier Ad Hoc Networks, 2003,1(4):351-369.

[2] Varadharajan V, Shankaran R. Security for Cluster-Based Ad Hoc Networks[J].Comp Commun., 2004,27(5): 488-501.

[3] Sakarindr P, Ansari N. Adaptive Trust-Based Anonymous Network [J]. Computer and Network Security, 2007,2(1):11-26.

[4] CHITI F, FANTACCI R. A broadband wireless communications system for emergency management[J]. IEEE Wireless Communications, 2008, 7(6): 8-14. 